\title{
AN ADDITIONAL CRITERION FOR THE FIRST CASE OF FERMAT'S LAST THEOREM ${ }^{1}$
}

\author{
BARKLEY ROSSER
}

In an earlier paper ${ }^{2}$ it was shown that if $p$ is an odd prime and

$$
a^{p}+b^{p}+c^{p}=0
$$

has a solution in integers prime to $p$, then

$$
m^{p-1} \equiv 1\left(\bmod p^{2}\right)
$$

for each prime $m \leqq 41$. In this paper the result is extended to $m \leqq 43$.

We will use the notations and conventions of I throughout, and a reference to a numbered equation will refer to the equation of that number in I. With $p$ assumed to be an odd prime such that (1) has a solution in integers prime to $p$, we assume that a $t$ exists such that the values of (2) satisfy (4), (5), and (6) with $m=43$. Put $g(x)=f(x) f(-x)$ and

$$
h(x)=\left(x^{42}-1\right) /\left(x^{6}-1\right) .
$$

Then $g(x)$ divides $h(x)$, and $g(x)$ can be completely factored modulo $p$.

Case 1. Assume that a root of $g(x)$ is a root of

$$
h(x) /\left(x^{12}+x^{10}+x^{8}+x^{6}+x^{4}+x^{2}+1\right) .
$$

Then this root belongs to either the exponent 21 or the exponent 42 modulo $p$. Hence $p \equiv 1(\bmod 42)$. So there is an $\omega$ such that

$$
\omega^{2}+\omega+1 \equiv 0 \text {. }
$$

Then $g(x), g(\omega x)$, and $g\left(\omega^{2} x\right)$ all divide $h(x)$. Moreover, the only cases in which two of $g(x), g(\omega x)$, and $g\left(\omega^{2} x\right)$ have a common factor are

I. $a^{6}+1 \equiv 0$,

II. $a^{6}+a^{3}+3 a^{2}+3 a+1 \equiv 0$,

III. $a^{6}-a^{3}-3 a^{2}-3 a-1 \equiv 0$,

or cases derived from these by replacing $a$ by one of the other roots of $f(x)$. So if we show that $h(x)$ has no factor in common with any of $x^{6}+1, x^{6}+x^{3}+3 x^{2}+3 x+1$, or $x^{6}-x^{3}-3 x^{2}-3 x-1$, then we can conclude that $g(x) g(\omega x) g\left(\omega^{2} x\right)$ must divide $h(x)$.

Clearly $h(x)$ has no factor in common with $x^{6}+1$.

1 Presented to the Society, April 27, 1940.

${ }^{2} A$ new lower bound for the exponent in the first case of Fermat's last theorem, this Bulletin, vol. 46 (1940), pp. 299-304. This paper will be referred to as I. 
Suppose $h(x)$ has a factor in common with $x^{6}+x^{3}+3 x^{2}+3 x+1$. This latter has the factors $x^{2}+x+1$ and $x^{4}-x^{3}+2 x+1$. The first has no factor in common with $h(x)$, since it divides $x^{6}-1$, which has no factor in common with $h(x)$. To test the second, we try it successively with each of the four factors of $h(x)$, getting the eliminants

$$
13 \cdot 19^{2} \cdot 127 \cdot 163^{2}, 5 \cdot 36913,2 \cdot 127,5 \cdot 7 \text {. }
$$

Suppose $h(x)$ has a factor in common with $x^{6}-x^{3}-3 x^{2}-3 x-1$. This latter has the factors $x^{2}-x-1$ and $x^{4}+x^{3}+2 x^{2}+2 x+1$. The first has no factor in common with $h(x)$ by Lemma 3 of I. Trying the second factor successively with each of the four factors of $h(x)$, we get the eliminants

$$
7^{3} \cdot 43,2^{2} \cdot 7 \cdot 13 \cdot 43,7,43 .
$$

So $g(x) g(\omega x) g\left(\omega^{2} x\right)$ must divide $h(x)$. Since both are of degree 36 , they must be equal. Putting $b=c+5$ and equating coefficients, we get

$$
\begin{aligned}
& A+1=2 c^{3}+3 c^{2}-24 c+13 \equiv 1, \\
& B+1=c^{6}+12 c^{5}+42 c^{4}+18 c^{3}-9 c^{2}-222 c+173 \equiv 1, \\
& C+1=-2 c^{6}+12 c^{5}+171 c^{4}+132 c^{3}-666 c^{2}+132 c+201 \equiv 1 .
\end{aligned}
$$

Dividing $16 B$ and $8 C$ by $A$, we get the remainder

$$
43 D=43\left(99 c^{2}+192 c-116\right) \equiv 0
$$

from each. Then

$$
2 c E=29 A+3 D=2 c\left(29 c^{2}+192 c-60\right) \equiv 0 .
$$

As $c \equiv 0$ would give $A \equiv 12 \equiv 0$, we have

$$
\begin{aligned}
28 c F=15 D-29 E & =28 c(23 c-96) \equiv 0, \\
29 c G=8 E-5 F & =29 c(8 c-49) \equiv 0, \\
8 F-23 G & =359 \equiv 0 .
\end{aligned}
$$

Case 2. Assume that no root of $g(x)$ is a root of

$$
h(x) /\left(x^{12}+x^{10}+x^{8}+x^{6}+x^{4}+x^{2}+1\right) .
$$

Then, since $g(x)$ divides $h(x)$ and is of degree 12,

$$
g(x) \equiv x^{12}+x^{10}+x^{8}+x^{6}+x^{4}+x^{2}+1 .
$$

So $2 c+1 \equiv 1$ and $c^{2}+5 \equiv 1$.

Princeton University 